\title{
Abnormal Resting State fMRI Activity Predicts Processing Speed Deficits in First-Episode Psychosis
}

\author{
Miklos Argyelan*, 1,2 , Juan A Gallego ${ }^{1,2,3}$, Delbert G Robinson ${ }^{1,2,3}$, Toshikazu Ikuta ${ }^{4}$, Deepak Sarpal ${ }^{1,2}$, \\ Majnu John ${ }^{1,2,5}$, Peter B Kingsley ${ }^{6}$, John Kane ${ }^{1,2,3}$, Anil K Malhotra ${ }^{1,2,3}$ and Philip R Szeszko ${ }^{1,2,3}$ \\ 'Center for Psychiatric Neuroscience, The Feinstein Institute for Medical Research, Manhasset, NY, USA; ${ }^{2}$ Division of Psychiatry Research, Zucker \\ Hillside Hospital, North Shore-LIl Health System, Glen Oaks, NY, USA; ${ }^{3}$ Departments of Psychiatry and Molecular Medicine, Hofstra North \\ Shore-LII School of Medicine, Hempstead, NY, USA; ${ }^{4}$ Department of Communication Sciences and Disorders, School of Applied Sciences, \\ University of Mississippi, University, MS, USA; ${ }^{5}$ Department of Mathematics, Hofstra University, Hempstead, NY, USA; ' ${ }^{6}$ epartment of \\ Radiology, North Shore University Hospital, Manhasset, NY, USA
}

Little is known regarding the neuropsychological significance of resting state functional magnetic resonance imaging (rs-fMRI) activity early in the course of psychosis. Moreover, no studies have used different approaches for analysis of rs-fMRI activity and examined gray matter thickness in the same cohort. In this study, 4 I patients experiencing a first-episode of psychosis (including $N=17$ who were antipsychotic drug-naive at the time of scanning) and $4 \mathrm{I}$ individually age- and sex-matched healthy volunteers completed rs-fMRI and structural MRI exams and neuropsychological assessments. We computed correlation matrices for 266 regions-of-interest across the brain to assess global connectivity. In addition, independent component analysis (ICA) was used to assess group differences in the expression of rs-fMRI activity within 20 predefined publicly available templates. Patients demonstrated lower overall rs-fMRI global connectivity compared with healthy volunteers without associated group differences in gray matter thickness assessed within the same regions-of-interest used in this analysis. Similarly, ICA revealed worse rs-fMRI expression scores across all 20 networks in patients compared with healthy volunteers, with posthoc analyses revealing significant ( $p<0.05$; corrected) abnormalities within the caudate nucleus and planum temporale. Worse processing speed correlated significantly with overall lower global connectivity using the region-of-interest approach and lower expression scores within the planum temporale using ICA. Our findings implicate dysfunction in rs-fMRI activity in first-episode psychosis prior to extensive antipsychotic treatment using different analytic approaches (in the absence of concomitant gray matter structural differences) that predict processing speed.

Neuropsychopharmacology (20 I5) 40, I631-1639; doi:10.1038/npp.2015.7; published online I8 February 2015

\section{INTRODUCTION}

Brain dysconnectivity has been hypothesized to have a central role in the neurobiology of schizophrenia (Friston and Frith, 1995; Pettersson-Yeo et al, 2011), with resting state functional magnetic resonance imaging (rs-fMRI) being used increasingly to characterize these abnormal networks. Several prior rs-fMRI studies conducted in patients with chronic schizophrenia reported lower overall activity (Alonso-Solís et al, 2012; Argyelan et al, 2014; Liang et al, 2006; Liu et al, 2006) as well as aberrant activity within language (Liemburg et al, 2012) and default mode/executive control networks (Hoptman et al, 2010; Woodward et al, 2012), disrupted connectivity between cortical and subcortical regions (Anticevic et al, 2014; Zhang et al, 2012;

\footnotetext{
*Correspondence: Dr M Argyelan, Division of Psychiatry Research, Zucker Hillside Hospital, North Shore-LII Health System, 75-59 263rd Street, Glen Oaks, NY II004, USA, Tel: +1 7184708175 , Fax: + 718343 1659, E-mail: margyela@nshs.edu

Received 25 July 2014; revised 18 December 2014; accepted 19 December 20।4; accepted article preview online 8 January 2015
}

Zhou et al, 2007) and interhemispheric dysconnectivity (Hoptman et al, 2012). It is widely acknowledged, however, that there are multiple ways to analyze rs-fMRI data. The use of different methods may contribute to inconsistent findings, and thus studies incorporating multiple approaches for rs-fMRI data analysis may shed light on abnormal circuits in the neurobiology of psychosis. Moreover, given that antipsychotics may potentially influence rs-fMRI measures (Lui et al, 2010), studies conducted early in the course of illness prior to extensive pharmacological intervention are of critical importance to the field, but findings have thus far been inconsistent (eg, Guo et al, 2014a,b; He et al, 2013; Lui et al, 2009, 2010; Ren et al, 2013).

Despite the widespread use of rs-fMRI studies in schizophrenia, there is still a paucity of work investigating the relationship between these deficits and neuropsychological functioning (Brennan et al, 2013). Bassett et al, (2012) reported that functional connectivity and topological metrics were correlated with verbal fluency performance and that network organization, which contained mainly weak connections, was correlated strongly with attention and memory in patients. In addition, variability within 
prefrontal and nonprefrontal connectivity (Cole et al, 2011) and altered connectivity between the frontal-parietal and cerebellar regions (Repovs et al, 2011) predicted severity of cognitive deficits in patients. He et al, (2012) reported that rs-fMRI abnormalities within the bilateral orbital frontal cortex were associated with cognitive processing speed in schizophrenia patients. These findings converge with prior work indicating that abnormal rs-fMRI connectivity was associated with worse processing speed in patients with chronic schizophrenia (Argyelan et al, 2014; Wang et al, 2014).

The investigation of gray matter structural abnormalities, which have been identified early in the course of illness (Shepherd et al, 2012), and rs-fMRI activity in the same cohort of patients is of critical importance to the interpretation of rs-fMRI studies, but little work has addressed this topic. Ren et al, (2013) reported group differences in gray matter volume mainly in thalamo-cortical networks, while alterations in the amplitude of low-frequency fluctuations were observed in fronto-parietal and default mode networks. In contrast, using voxel-based morphometry and rs-fMRI Lui et al, (2009) reported no rs-fMRI connectivity abnormalities within brain regions with less gray matter in patients compared with healthy volunteers, including the superior temporal gyrus, middle temporal gyrus, and anterior cingulate gyrus. Although voxel-based morphometry and voxel-based cortical thickness can provide similar results, the former may be influenced by cortical surface folding, and prior work has attributed greater sensitivity to voxel-based cortical thickness measures (Hutton et al, 2009), which we thus used in the current study.

The goals of the current study were (1) to examine rsfMRI activity in patients experiencing a first-episode of psychosis with minimal or no prior antipsychotic treatment compared with healthy volunteers using region-of-interest analyses encompassing 266 cortical and subcortical regions and ICA using 20 predefined templates of brain network activity; (2) to examine the functional correlates of these rs-fMRI patterns; and (3) to investigate the relationship between rs-fMRI activity and cortical thickness within the same regions-of-interest. We hypothesized that patients would demonstrate global abnormalities in rs-fMRI activity using both region-of-interest and ICA approaches and that these functional abnormalities would be associated with worse processing speed and greater clinical impairment consistent with our prior study in chronic schizophrenia.

\section{MATERIALS AND METHODS}

\section{Subjects}

Forty-one patients experiencing a first-episode of psychosis were recruited from admissions to the Zucker Hillside Hospital as part of their participation in an NIMHsponsored, 52-week, double-blind, randomized, controlled trial (ClinicalTrials.gov ID: NCT00320671). Patients received a physical exam and laboratory screening to rule out medical causes for their psychotic episode. Patient diagnoses were based on the Structured Clinical Interview for Axis I DSM-IV Disorders (SCID) supplemented by information from clinicians and family members. Firstepisode patients met DSM-IV criteria for schizophrenia (undifferentiated $=17$ and paranoid $=10$ ), schizophreniform disorder $(n=8)$, schizoaffective disorder $(N=1)$, or psychosis not otherwise specified $(N=5)$. Seventeen patients met DSM criteria for substance use/dependence diagnoses, including (numbers in parentheses): cannabis abuse/dependence $(N=16)$, alcohol abuse/dependence $(N=6)$, cocaine dependence $(N=2)$, amphetamine dependence $(N=1)$, and polysubstance dependence $(N=1)$. On average, patients had a total lifetime exposure of 5.1 (median $=4.0$; range $=$ $0-21)$ days of antipsychotic treatment prior to the scan. The minimally treated patients were (1) treated briefly in the clinical trial prior to the baseline scan; (2) untreated at the time of the baseline scan, but had prior exposure to antipsychotics previously; or (3) both. Seventeen patients were antipsychotic drug-naive at the time of the scan. Mean age of patients were 21.5 years $(\mathrm{SD}=5.5)$. Mean age at first psychotic symptoms was 19.0 years $(S D=5.6)$; data were unavailable for three patients.

We recruited 41 healthy volunteers from advertisements posted on websites and by word of mouth. Exclusion criteria for healthy volunteers included the lifetime history of a major mood or psychotic disorder as determined by clinical interview using the SCID-NP. Exclusion criteria for all study participants included MRI contraindications, serious medical conditions, or mental retardation. This study was approved by the NSLIJ IRB, and written informed consent was obtained from all study participants or their parents in the case of minors. All minors provided written informed assent to participate in the study.

\section{Image Acquisition}

MRI exams were conducted at NSUH on a 3T GE HDx scanner. For image registration, we acquired anatomical scans in the coronal plane using a 3D spoiled gradient (SPGR) sequence $(\mathrm{TR}=7.5 \mathrm{~ms}, \mathrm{TE}=3 \mathrm{~ms}$, matrix $=256 \times 256, \quad \mathrm{FOV}=240$ $\mathrm{mm}$ ) producing 216 contiguous images (slice thickness $=1$ $\mathrm{mm}$ ) through the whole head. We acquired rs-fMRI scans comprising a total of 150 echo-planner imaging volumes with the following parameters: $\mathrm{TR}=2000 \mathrm{~ms}, \mathrm{TE}=30 \mathrm{~ms}$, matrix $=64^{\star} 64, \mathrm{FOV}=240 \mathrm{~mm}$, slice thickness $=3 \mathrm{~mm}$, and 40 continuous axial oblique slices (one voxel $=$ $3.75 \times 3.75 \times 3 \mathrm{~mm}^{3}$ ). During the acquisition, all subjects were instructed to 'close their eyes and not think of anything in particular.'

\section{Motion Analyses}

We investigated the potential effects of motion on functional connectivity by examining both relative and absolute motion displacement during the rs-fMRI exam (see Supplementary Material)

\section{Image Processing}

We used FSL (http://www.fmrib.ox.ac.uk) and AFNI (http:// afni.nimh.nih.gov/afni) based script libraries from the 1000 Functional Connectomes Project (http://www.nitrc.org/ projects/fcon_1000 (Biswal et al, 2010) for preprocessing and a lab-developed script in the R statistical language for additional analysis as described below. All resting-state scans were preprocessed using the scripts from the 1000 
Functional Connectomes Project ('fcon scripts'). Standard preprocessing included removal of the first four 'dummy' scans, motion correction, and spatial smoothing (6-mm FWHM Gaussian kernel). Standard registration and normalization to MNI152 space was employed with the resulting transformation applied to each individual's functional data set (12 parameter affine transformation). The time series were then high- and low-pass filtered (cutoff frequencies were 0.005 and $0.1 \mathrm{~Hz}$, respectively). Each individual's 4D time series data were regressed on eight predictors: white matter (WM), cerebrospinal fluid, and six motion parameters. Consistent with our prior work (Argyelan et al, 2014) and others (Yang et al, 2014), we did not regress out global signal, because it would have interfered with the connectivity strength (CS) calculation.

\section{Image Processing Using Regional CS}

We computed regional mean time series for all individuals' rs-fMRI data by using a set of predefined regions (Power et al, 2011) used in chronic psychosis patients previously (Argyelan et al, 2014). In their prior study, Power et al, (2011) demonstrated that their 264 regions are not only functionally relevant but can eliminate artificial low distance correlations. We also added caudate nucleus regions-ofinterest bilaterally. After obtaining 266 time series per subject, we decomposed these signals with maximal overlap discrete wavelet transform (Percival and Walden, 2000) similar to the method described by Lynall et al, (2010). We used the Daubechies wavelet transform filter of length 4 and used the $0.060-0.125 \mathrm{~Hz}$ (due to the preprocessing cutoff frequency at $0.1 \mathrm{~Hz}$; in our case, this covers the range of $0.06-0.10 \mathrm{~Hz}$ ) scale wavelet coefficients for further analysis. We used the $\mathrm{R}$ wavelet package to implement these calculations (Aldrich, 2013). We then estimated the correlation of these wavelet transformed signals (wavelet coefficients) between each possible pair of regions. For each $i$ region, we then averaged the correlation coefficients that $i$ region had with all the other $j$ regions (ie, we averaged each row of the correlation matrix), and according to its usual name in the connectivity literature, we called this metric CS. This measure reflects how strongly one region is connected to other regions in general and is called regionalbased CS. Global connectivity was defined as the first principal component of the variance across these measures (Argyelan et al, 2014). Repeated-measures analysis of variance was used to examine whether regions-of-interest were significantly different between groups in the global connectivity analysis. The within-subject factor was regionof-interest $(n=266)$, and the between-subject factor was the group (patient $v s$ control).

\section{Image Processing Using Independent Component Analysis (ICA)}

To further support our analysis with regional-based connectivity measures, we also conducted ICA. In 2010, Biswal et al, (2010) reported evidence for 20 independent rs-fMRI components within the brain making it feasible to examine patterns of resting state activation using publicly available templates and facilitate cross-study comparisons. In the current study, the dual-regression approach was used to identify within-subjects' fMRI data, subject-specific temporal dynamics and associated spatial maps to compute 'expression scores' within these 20 predefined networks (Biswal et al, 2010). This involved using the full set of predefined group-ICA spatial maps in a linear model (ie, spatial regression) against our rs-fMRI data sets, resulting in matrices describing temporal dynamics for each component and individual. This was then followed by using the time course matrices in a linear model (ie, temporal regression) against the rs-fMRI data set to estimate subject-specific spatial maps. Individual spatial maps were correlated with the predefined healthy group ICA spatial templates to estimate the degree of 'expression' within 20 individual brain networks acquired in a large cohort of healthy volunteers (Biswal et al, 2010). Thus these individual network 'expression scores' were higher if that particular network's intra-network correlations conformed better to the standard network (which was determined using a large healthy data set). We used repeated-measures ANCOVA to determine how well the resting state data from our study were expressed within these 20 predefined networks. Network served as a within-subjects factor, whereas sex and group served as between-subjects factors. Age and intracranial volume served as statistical covariates. Independent components identified as significantly different between patients and healthy volunteers were subsequently examined using voxelwise analysis in SPM5 $(p<0.05$, FWE corrected) to identify regional differences within networks.

\section{Cortical Thickness Analysis}

We used FreeSurfer (v5.1) to conduct cortical reconstruction of our 3D MRI data set (http://surfer.nmr.mgh.harvard. edu). Using well-documented steps described previously (Dale et al, 1999; Fischl and Dale, 2000), a pial surface and a gray-WM interface was created and stored with a triangular tessellation representation. The distance between these surfaces, which is the cortical thickness, is calculated for each vertex of the triangular representation. Using a freesurfer script (mri_surf2vol), we projected our 266 nodes so that vertices were assigned with node numbers, and thus we were able to calculate the thickness scores associated with the individual nodes. This approach is illustrated in Figure 1. Repeated-measures analysis of variance was used to examine whether groups differed in cortical thickness with the within-subjects factor being region-of-interest. In addition, we used principal component analysis to determine whether this method could separate the groups from each other.

\section{Clinical, Neuropsychological, and Handedness Assessments}

Patients completed the 18-item Brief Psychiatric Rating Scale Anchored-version (Overall and Gorham, 1962), and we derived a total score by summing all the items. We also administered the MCCB to patients with our apriori hypothesis focused on processing speed. The neuropsychological domains and total BPRS score were investigated in relationship to the overall global connectivity metric and regions that distinguished patients from healthy volunteers in the voxelwise results from the IC analysis using Pearson 
a

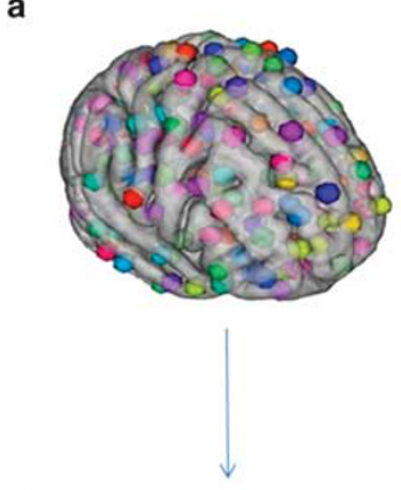

b

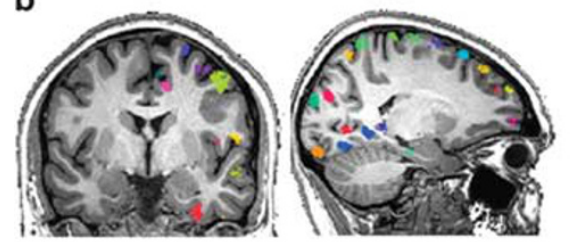

a
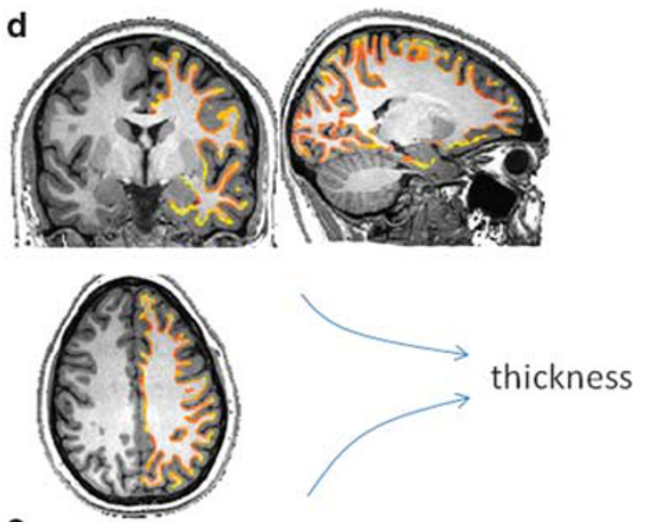

C
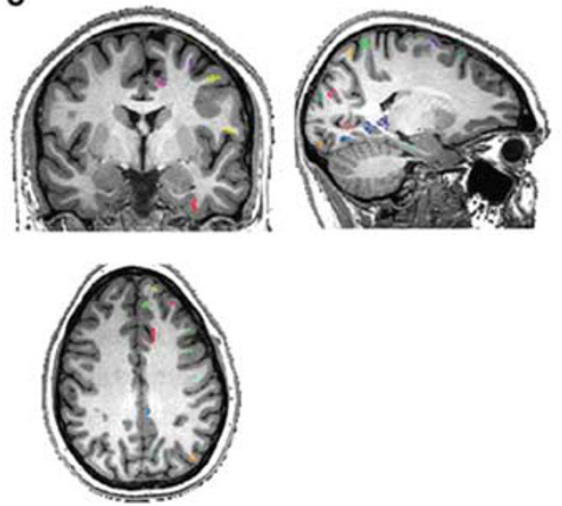

Figure I Region-based cortical thickness approach. The 266 nodes that were used as resting state functional magnetic resonance imaging seeds are illustrated in panel a. The nodes were then projected into Freesurfer space aligning them with vertices from the gray-white interface. The vertices with the corresponding gray matter ribbon are illustrated in the left hemisphere in panel b. Regions-of-interest and their corresponding gray-white matter interface are provided in panel c. The gray-white matter interface, color-coded with corresponding thickness is illustrated in panel d. Lighter colors denote thicker gray matter regions above that area such that gray matter is defined as the distance between the interface and the pial surface. The region-level calculation of thickness is an average of the regions in panel c over the values in panel $d$. This is illustrated in the left hemisphere but was done similarly in the right hemisphere.

Product Moment correlations. We minimized type-I error in structure-function analyses by adjusting alpha to 0.01 (two-tailed). All individuals were classified as either rightor left-handed based on a modified version of the Edinburgh Inventory.

\section{RESULTS}

The first-episode patient and healthy comparison groups were well matched on relevant demographic factors. Specifically, the groups did not differ significantly $(p<0.05)$ in distributions of age, sex, handedness, education, height, or weight (Table 1). In patients, the mean total BPRS score at baseline was $43.7 \pm 8.2$. A greater proportion of patients were smokers compared with healthy volunteers $(p=0.013$, $\mathrm{OR}=6.99$, Fisher's Exact Test). BPRS scores for antipsychotic drug-naive patients was $43.9(\mathrm{SD}=9.7)$ and for minimally treated patients was $43.5(\mathrm{SD}=7.2)$. There were no significant $(p<0.05)$ group differences in the distributions of average FD or DVARS values between groups as illustrated in Supplementary Figures S1 and S2.

Analysis of the global CS measure revealed a significant main effect of group $(\mathrm{F}=36.09, \mathrm{df}=1, p<0.001)$ such that global connectivity was significantly lower in patients compared with healthy volunteers overall (Figure 2), but there was no significant group $\times$ region interaction $(p>0.05)$. There was no significant $(p>0.05)$ difference in the global CS between antipsychotic drug-naive patients and those patients who had received minimal treatment. Patients who were smokers had greater connectivity compared with nonsmoking patients $(\mathrm{F}=42.49, \mathrm{df}=1, p<0.001$, Supplementary Figure S3). Substance use did not have any significant effect on functional connectivity among patients (Supplementary Figure S3). Greater global dysconnectivity correlated significantly with slower processing speed among patients $(r=-0.45, \mathrm{df}=37, p=0.005)$ but not with any of the other neuropsychological domains or BPRS total score.

The individual expression scores from the ICA for patients and healthy volunteers are provided in Figure 3. The results of this analysis revealed a significant main effect of group $(\mathrm{F}=16.68, \mathrm{df}=76, p<0.001)$, as well as a groupby-IC interaction $(\mathrm{F}=1.79, \mathrm{df}=76, p=0.049)$. Follow-up univariate ANCOVAs revealed that patients had significantly lower expression scores compared with healthy volunteers within the medial visual (IC1; $\mathrm{F}=15.03, \mathrm{df}=1,76$, $p<0.001$ ), lateral visual (IC3; $\mathrm{F}=7.59, \mathrm{df}=1,76, p=0.007$ ), posterior cingulate default mode (IC6; $\mathrm{F}=3.99, \mathrm{df}=1,76$, $p=0.049$ ), ventral default mode (IC14; $\mathrm{F}=9.53, \mathrm{df}=1,76$, $p=0.003$ ), salience (IC16; $\mathrm{F}=6.08, \mathrm{df}=1,76, p=0.016$ ), 
Table I Sample Characteristics

\begin{tabular}{|c|c|c|c|c|c|}
\hline & First-episode patients $(N=4 I)$ & Healthy volunteers $(N=4 I)$ & df & Test statistic & $p$-Value \\
\hline Age & $21.5(5.5)$ & $21.5(5.1)$ & 80 & $t=0.05$ & NS \\
\hline $\operatorname{Sex}(M / F)$ & $29 \mathrm{M} / \mathrm{I} 2 \mathrm{~F}$ & $29 \mathrm{M} / \mathrm{I} 2 \mathrm{~F}$ & । & $\chi^{2}=0$ & NS \\
\hline Laterality quotient & $0.77(0.33)$ & $0.69(0.49)$ & 80 & $t=-0.89$ & NS \\
\hline Education (years) & $12.2(2.1)$ & I3.3 (3.3) & 80 & $t=1.84$ & NS \\
\hline Height (inches) & $67.4(3.6)$ & $68.2(4.1)$ & 79 & $t=0.85$ & NS \\
\hline
\end{tabular}

Data are presented as mean \pm SD in parentheses, unless otherwise indicated. Height and weight were unavailable for one patient.

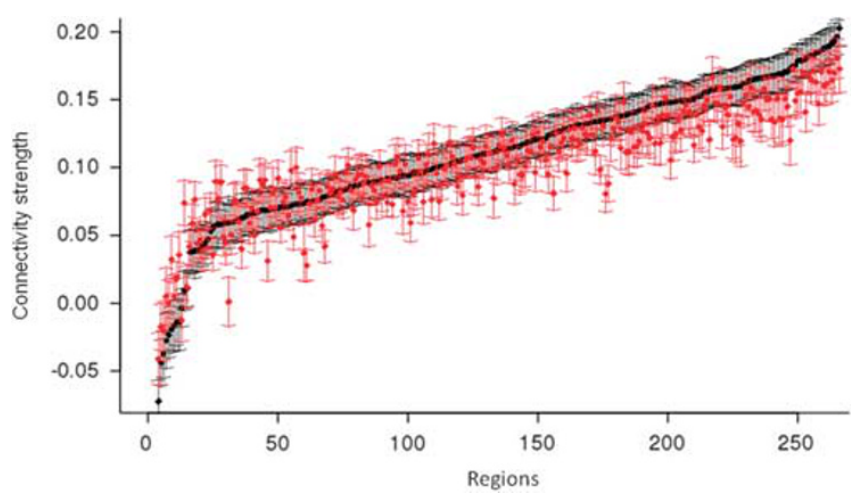

Figure 2 Average connectivity strength in patients and healthy volunteers. Red lines denote patients, and black lines denote healthy volunteers. SEMs are provided for each node. Regions are presented based on their connectivity strength rank order in healthy volunteers.

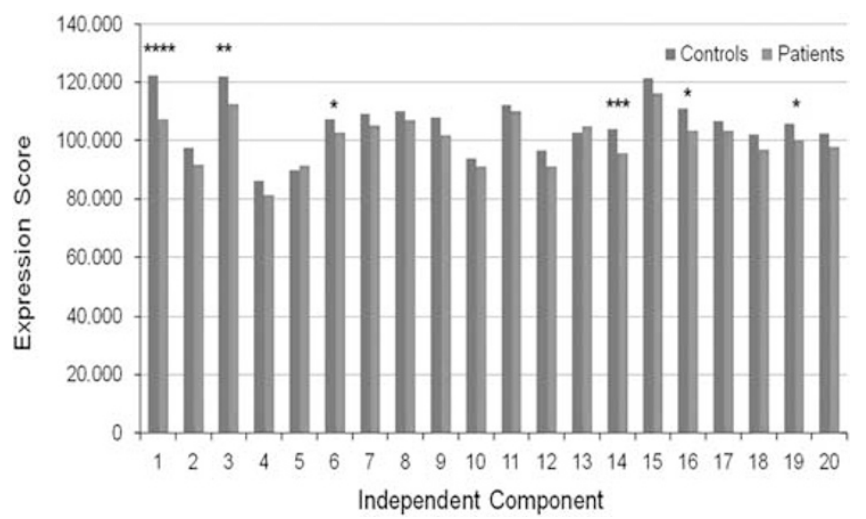

Figure 3 Independent component analysis expression scores in patients and healthy volunteers. ${ }^{*} p<0.05$; $* * * 0.0$ I; ${ }^{* * *} p<0.005$; $* * * * * 0.00$ I.

and somatosensory (IC19; $\mathrm{F}=6.07, \mathrm{df}=1,76, p=0.016$ ) networks. Between-group voxelwise analyses of these six ICs revealed significantly $(p<0.05$, FWE corrected) lower rsfMRI activity within the left planum temporale (MNI space, $x=-44, y=-32, z=10$; Figure $4 \mathrm{a}$ and $\mathrm{b}$ ) within the salience network (IC16) and the basal ganglia (MNI space, $x=-44, y=12, z=56$; Figure $4 \mathrm{c}$ and d) within the somatosensory network (IC19) in patients compared with healthy volunteers. An illustration of the four additional networks (ie, IC1, IC3, IC6, IC14) that differed between groups but did not yield significant voxelwise differences is provided in Supplementary Figure S4. Investigation of the neuropsychological correlates of IC16 and IC19 revealed that slower processing speed correlated with lower rs-fMRI activity within the left planum temporale $(\mathrm{r}=0.44, \mathrm{df}=37$, $p=0.007$ ) but not with any of the other neuropsychological domains or total BPRS score. There was no significant $(p>0.05)$ difference in the above discussed measures (CS, planum temporale, or basal ganglia rs-fMRI measures) between antipsychotic drug-naive patients and those patients who had received minimal treatment.

\section{Cortical Thickness Measures}

Analysis of the cortical thickness measures did not reveal either a significant group main effect or significant group $\times$ region interaction. Additional principal component analysis could not significantly differentiate the groups from each other. Moreover, cortical thickness did not correlate with CS assessed by the rs-fMRI measures.

\section{DISCUSSION}

In this study, we used complementary approaches for analysis of rs-fMRI data in patients experiencing a first episode of psychosis. We observed significantly lower overall rs-fMRI activity using a region-of-interest-based global CS metric as well as overall worse expression scores across 20 predefined templates using ICA in patients compared with healthy volunteers. Moreover, both analytic approaches provide evidence for a relationship between abnormal rs-fMRI activity and processing speed deficits. Resting state fMRI abnormalities in our first-episode patients were observed in the absence of overall or regionally specific gray matter thickness differences between groups. Strengths of our study include the use of a well-characterized clinical cohort of first-episode psychosis patients studied early in the course of psychosis prior to extensive pharmacological intervention, use of healthy volunteers individually age- and sex-matched to patients, different analytic approaches of the rs-fMRI data, investigation of neuropsychological functions in relationship to rs-fMRI activity in patients and use of the same regions-of-interest to investigate cortical thickness and rs-fMRI measures.

The results of the region-of-interest global connectivity analysis replicate our prior findings in chronic schizophrenia (Argyelan et al, 2014) using a different cohort of individuals with the same methods and sequence parameters 

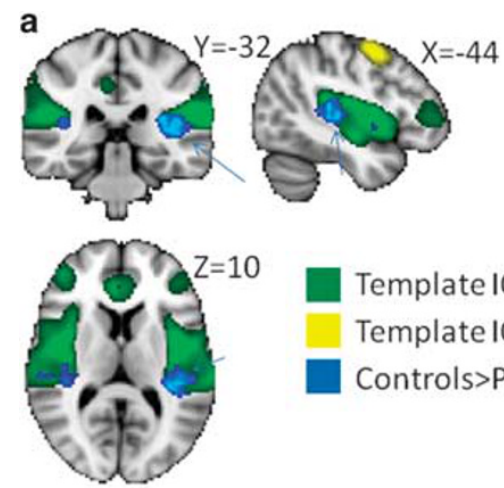

TemplateIC16+ Template IC 16 Controls $>$ Patients
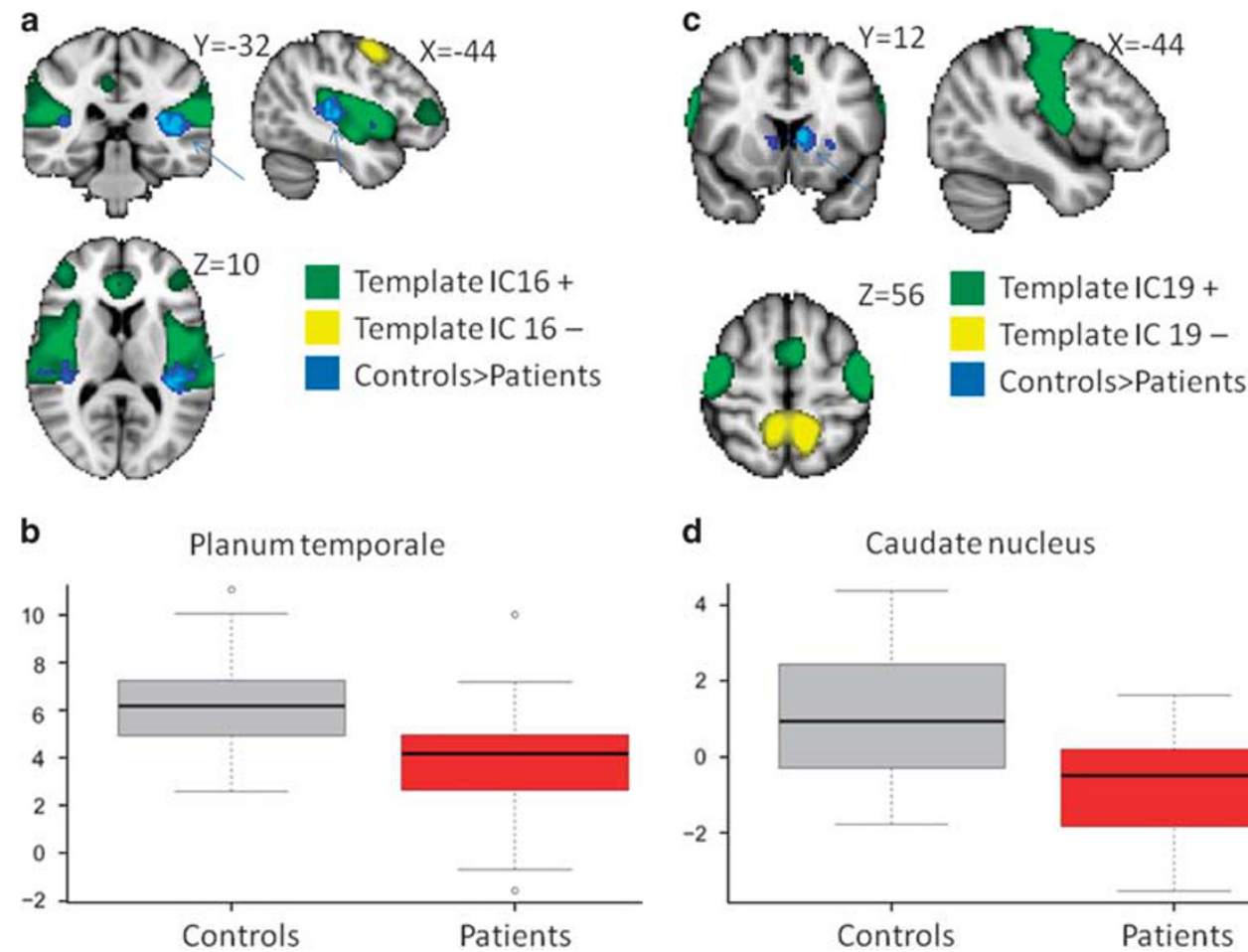

d

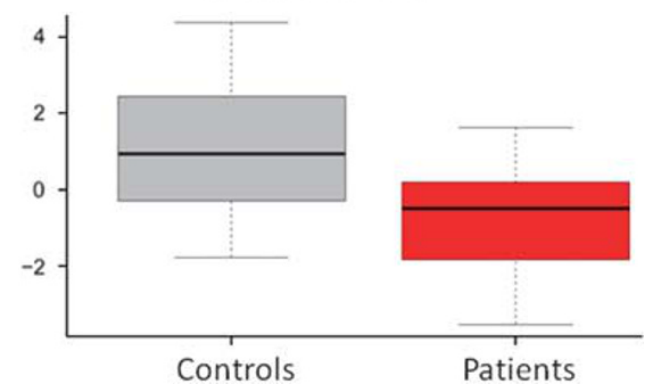

Figure 4 Illustration of significant ( $p<0.05$; FWE-corrected) voxelwise analyses of independent components 16 and 19. Green and yellow indicate strongly correlating and anti-correlating regions respectively. (a) Salience network, blue indicates group differences in planum temporale, (c) Somatosensory network, blue indicates group differences in caudate nucleus, (b) and (d) are post hoc analyses in the corresponding blue region.

as the current study. In contrast to our prior study, however, we did not observe rs-fMRI connectivity abnormalities within specific regions-of-interest in patients compared with healthy volunteers. Coupled together, these studies are consistent with the possibility that although global dysconnectivity may be evident in both first-episode and chronic patients, there may be regionally specific changes in function over time that may involve striatal and/ or thalamic regions (as observed in our prior study of chronic patients) that could conceivably be related to illness and/or medication factors. Moreover, consistent with our prior study in chronic patients (Argyelan et al, 2014) we also observed significant abnormalities in first-episode patients compared with healthy volunteers in sensory networks (both visual, auditory, and somatosensory). This may be particularly noteworthy because both prior empirical and theoretical work implicate a disruption in perceptual processes in schizophrenia (Butler et al, 2001; Grillon et al, 1992; Javitt et al, 1996; Notredame et al, 2014; Patterson et al, 2008; Umbricht and Krljes, 2005).

Consistent with the global connectivity analysis, we identified lower expression scores in first-episode patients overall compared with healthy volunteers using ICA. In contrast to the global connectivity analysis, however, the ICA implicated dysfunction within regions comprising the salience network, and in particular, the planum temporal. Brain regions comprising the salience network, including the temporal lobe, are critical for recruiting other networks to process environmental and sensory stimuli, have a role in self-monitoring and agency and have been strongly implicated in the neurobiology of psychosis (White et al, 2013). Specifically, increasing evidence suggests that ab- normal salience contributes to the emergence of psychotic symptoms through dopamine dysregulation leading individuals to attribute environmental significance to stimuli that are otherwise considered irrelevant (Howes and Kapur, 2009). Aberrant salience processing may underlie psychotic symptoms and involve functional alterations in the striatum, hippocampus, and other subcortical dopamine regions in association with temporal regions (Orliac et al, 2013; Roiser et al, 2013). Other investigators (Heinz and Schlagenhauf, 2010) highlight that dopamine dysregulation in patients with psychosis could lead to aberrant attribution of incentive salience, thus leading to the emergence of psychotic behavior. Although we did not observe functional differences between antipsychotic drug-naive and minimally treated patients in this study, it is important to acknowledge that antipsychotic treatment often requires weeks to demonstrate efficacy, and thus our findings are not inconsistent with the salience network serving as either a state- or trait-related phenomenon associated with the emergence of psychotic symptoms.

Using ICA, we also observed lower rs-fMRI activity within the caudate nucleus in first-episode patients compared with healthy volunteers. These findings suggest that functional connectivity between the caudate nucleus and cortical networks is impaired in schizophrenia. Indeed, other studies (Hoptman et al, 2010; Turner et al, 2013) implicated rs-fMRI abnormalities within the caudate nucleus and reported that an increase in the low frequency fluctuations in these areas may be associated with successful antipsychotic treatment (Lui et al, 2010). It should be acknowledged that while the caudate demonstrated lower connectivity in the ICA analysis in patients, it was outside 
the network investigated. This therefore indicates that, contrary to IC 16 where intra-network changes were associated with group differences, in this network (IC 19) the source of group difference was mainly due to internetwork disruption. Given that inter-network correlations tend to be much weaker compared with intra-network correlations, these results indicate that these correlations could nonetheless result in significant group differences.

The finding that lower rs-fMRI global connectivity was associated with worse processing speed replicates our prior work in chronic patients (Argyelan et al, 2014) and thus extends this relationship to first-episode psychosis. Only processing speed correlated with aberrant rs-fMRI activity within the salience network (including the planum temporale) using ICA. This finding converges with increasing evidence that visuomotor coordination and motor representation are modulated by neurons within the temporal region (Tankus and Fried, 2012) and that cognitive processing speed is associated with temporal cortical structural integrity (Turken et al, 2008). Moreover, some data suggest that higher stages of language processing operate at a fixed speed and could theoretically impose a 'temporal bottleneck' on language functions (Vagharchakian et al, 2012). In this regard, Takeuchi et al, (2011) demonstrated that processing speed training was associated with both structural and functional changes in temporal regions.

It may be noteworthy that processing speed deficits were associated with rs-fMRI abnormalities as these neuropsychological deficits are often the most pronounced in firstepisode schizophrenia as observed in meta-analytic studies (Mesholam-Gately et al, 2009). Thus processing speed may be particularly good in capturing generalized dysfunction contributing to widespread cognitive failures in schizophrenia (Rodríguez-Sánchez et al, 2007). In addition, processing speed deficits have been observed in high-risk samples compared with healthy individuals (Seidman et al, 2010), at-risk subjects who converted to psychosis (Lin et al, 2011), and adolescents in the community who are prodromal for psychosis (Kelleher et al, 2013). There are also data indicating that poor social outcome can be predicted by reduced processing speed in a clinical high-risk sample for psychosis (Carrión et al, 2013; Faber et al, 2011). Given that processing speed deficits are present in first-degree relatives of patients with schizophrenia ( $\mathrm{Ma}$ et al, 2007), such measures could represent a valuable endophenotype, in combination with rs-fMRI studies, for molecular genetic studies of schizophrenia.

There are several limitations to our study that should be acknowledged. Although we did not observe group differences in cortical thickness measures assessed using the same regions-of-interest as the rsfMRI analysis, this does not preclude the possibility that group differences would be evident using larger regions-of-interest or even volumetric (manual or semi-automated) approaches. In addition, the use of global connectivity measures with 266 nodes could miss more focused abnormalities. An additional limitation may be the instructions provided to the subjects in that 'eyes open' $v s$ 'eyes closed' have yielded different findings in the literature (Xu et al, 2014; Patriat et al, 2013), although in this study all subjects were provided with the same instructions. An additional limitation is that our findings may be influenced by factors such as substance use or smoking history. Ancillary analyses, however, indicated that functional connectivity was not significantly different among patients with and without a substance use history (Supplementary Figure S3). Moreover, in the case of smoking, the observed influence was in the opposite direction (ie, smoking history was associated with greater functional connectivity; Supplementary Figure S3). We also acknowledge that even a very short duration of antipsychotic treatment can have a significant effect on functional connectivity (Cole et al, 2013); however, our ancillary analyses did not indicate any differences between drugnaive and minimally treated patients.

In sum, our findings suggest that rs-fMRI abnormalities are evident in patients experiencing a first episode of psychosis prior to extensive pharmacological intervention assessed using both a global connectivity metric and ICA in the absence of concomitant gray matter structural alterations and that these rs-fMRI abnormalities predict processing speed deficits.

\section{FUNDING AND DISCLOSURE}

This work was supported in part by grants from NARSAD (PRS) and the National Institute of Mental Health to Dr Szeszko (R01 MH076995) and Dr Robinson (R01 MH060004), the NSLIJ Research Institute General Clinical Research Center (M01 RR018535), an Advanced Center for Intervention and Services Research (P30 MH090590) and a Center for Intervention Development and Applied Research (P50 MH080173). Dr Robinson has been a consultant to Asubio and Shire, and he has received grants from BristolMeyers Squibb, Janssen, and Otsuka. Dr Kane is a shareholder in Medvante, Inc., has been a consultant for Amgen, Alkermes, Bristol-Meyers Squibb, Eli Lilly, Forrest Pharmaceuticals, Genentech, H. Lundbeck Intracellular Therapeutics, Janssen Pharmaceutica, Jazz Pharmaceuticals, Johnson and Johnson, Lundbeck, Merck, Novartis, Otsuka, Pierre Fabre, Proteus, Reviva, Roche, and Sunovion, and has been on the Speaker's Bureau for Bristol-Meyers Squibb, Eli Lilly, Janssen, and Otsuka. Dr Malhotra is a consultant to Genomind, Inc. Dr Szeszko has received grant support from Otsuka. Dr Sarpal has received research support from Janssen Pharmaceutica. This support is unrelated to the accepted manuscript. The other authors report no financial relationships with commercial interests.

\section{ACKNOWLEDGMENTS}

We appreciate the constructive comments of the anonymous reviewers in an earlier version of this manuscript.

\section{REFERENCES}

Aldrich E (2013): Wavelets: A package of functions for computing wavelet filters, wavelet transforms and multiresolution analyses. Available at <http://cran.r-project.org/web/packages/wavelets/ index.html $>$.

Alonso-Solís A, Corripio I, de Castro-Manglano P, Duran-Sindreu S, Garcia-Garcia M, Proal E et al (2012). Altered default network resting state functional connectivity in patients with a first episode of psychosis. Schizophr Res 139: 13-18. 
Anticevic A, Cole MW, Repovs G, Murray JD, Brumbaugh MS, Winkler AM et al (2014). Characterizing thalamo-cortical disturbances in schizophrenia and bipolar illness. Cereb Cortex 24: 3116-3130.

Argyelan M, Ikuta T, DeRosse P, Braga RJ, Burdick KE, John M et al (2014). Resting-state FMRI connectivity impairment in schizophrenia and bipolar disorder. Schizophr Bull 40: 100-110.

Bassett DS, Nelson BG, Mueller BA, Camchong J, Lim KO (2012). Altered resting state complexity in schizophrenia. Neuroimage 59: 2196-2207.

Biswal BB, Mennes M, Zuo X-N, Gohel S, Kelly C, Smith SM et al (2010). Toward discovery science of human brain function. Proc Natl Acad Sci USA 107: 4734-4739.

Brennan AM, Harris AWF, Williams LM (2013). Functional dysconnectivity in schizophrenia and its relationship to neural synchrony. Expert Rev Neurother 13: 755-765.

Butler PD, Schechter I, Zemon V, Schwartz SG, Greenstein VC, Gordon J et al (2001). Dysfunction of early-stage visual processing in schizophrenia. Am J Psychiatry 158: 1126-1133.

Carrión RE, McLaughlin D, Goldberg TE, Auther AM, Olsen RH, Olvet DM et al (2013). Prediction of functional outcome in individuals at clinical high risk for psychosis. JAMA Psychiatry 70: $1133-1142$.

Cole DM, Oei NYL, Soeter RP, Both S, van Gerven JMA, Rombouts SARB et al (2013). Dopamine-dependent architecture of corticosubcortical network connectivity. Cereb Cortex 23: 1509-1516.

Cole MW, Anticevic A, Repovs G, Barch D (2011). Variable global dysconnectivity and individual differences in schizophrenia. Biol Psychiatry 70: 43-50.

Dale AM, Fischl B, Sereno MI (1999). Cortical surface-based analysis. I. Segmentation and surface reconstruction. Neuroimage 9: 179-194.

Faber G, Smid HGOM, Van Gool AR, Wunderink L, Wiersma D, van den Bosch RJ (2011). Neurocognition and recovery in first episode psychosis. Psychiatry Res 188: 1-6.

Fischl B, Dale AM (2000). Measuring the thickness of the human cerebral cortex from magnetic resonance images. Proc Natl Acad Sci USA 97: 11050-11055.

Friston KJ, Frith CD (1995). Schizophrenia: a disconnection syndrome? Clin Neurosci 3: 89-97.

Grillon C, Ameli R, Charney DS, Krystal J, Braff D (1992). Startle gating deficits occur across prepulse intensities in schizophrenic patients. Biol Psychiatry 32: 939-943.

Guo W, Xiao C, Liu G, Wooderson SC, Zhang Z, Zhang J et al (2014a). Decreased resting-state interhemispheric coordination in first-episode, drug-naive paranoid schizophrenia. Prog Neuropsychopharmacol Biol Psychiatry 48: 14-19.

Guo W, Yao D, Jiang J, Su Q, Zhang Z, Zhang J et al (2014b). Abnormal default-mode network homogeneity in first-episode, drug-naive schizophrenia at rest. Prog Neuropsychopharmacol Biol Psychiatry 49: 16-20.

He H, Sui J, Yu Q, Turner JA, Ho B-C, Sponheim SR et al (2012). Altered small-world brain networks in schizophrenia patients during working memory performance. PLoS One 7: e38195.

He Z, Deng W, Li M, Chen Z, Jiang L, Wang Q et al (2013). Aberrant intrinsic brain activity and cognitive deficit in firstepisode treatment-naive patients with schizophrenia. Psychol Med 43: 769-780.

Heinz A, Schlagenhauf F (2010). Dopaminergic dysfunction in schizophrenia: salience attribution revisited. Schizophr Bull 36: $472-485$.

Hoptman MJ, Zuo X-N, Butler PD, Javitt DC, D’Angelo D, Mauro CJ et al (2010). Amplitude of low-frequency oscillations in schizophrenia: a resting state fMRI study. Schizophr Res 117: 13-20.

Hoptman MJ, Zuo X-N, D’Angelo D, Mauro CJ, Butler PD, Milham MP et al (2012). Decreased interhemispheric coordination in schizophrenia: a resting state fMRI study. Schizophr Res 141: 1-7.
Howes OD, Kapur S (2009). The dopamine hypothesis of schizophrenia: version III-the final common pathway. Schizophr Bull 35: 549-562.

Hutton C, Draganski B, Ashburner J, Weiskopf N (2009). A comparison between voxel-based cortical thickness and voxel-based morphometry in normal aging. Neuroimage 48: 371-380.

Javitt DC, Steinschneider M, Schroeder CE, Arezzo JC (1996). Role of cortical N-methyl-D-aspartate receptors in auditory sensory memory and mismatch negativity generation: implications for schizophrenia. Proc Natl Acad Sci USA 93: 11962-11967.

Kelleher I, Clarke MC, Rawdon C, Murphy J, Cannon M (2013). Neurocognition in the extended psychosis phenotype: performance of a community sample of adolescents with psychotic symptoms on the MATRICS neurocognitive battery. Schizophr Bull 39: 1018-1026.

Liang M, Zhou Y, Jiang T, Liu Z, Tian L, Liu H et al (2006). Widespread functional disconnectivity in schizophrenia with resting-state functional magnetic resonance imaging. Neuroreport 17: 209-213.

Liemburg EJ, Vercammen A, Ter Horst GJ, Curcic-Blake B, Knegtering H, Aleman A (2012). Abnormal connectivity between attentional, language and auditory networks in schizophrenia. Schizophr Res 135: 15-22.

Lin A, Wood SJ, Nelson B, Brewer WJ, Spiliotacopoulos D, Bruxner A et al (2011). Neurocognitive predictors of functional outcome two to 13 years after identification as ultra-high risk for psychosis. Schizophr Res 132: 1-7.

Liu H, Liu Z, Liang M, Hao Y, Tan L, Kuang F et al (2006). Decreased regional homogeneity in schizophrenia: a resting state functional magnetic resonance imaging study. Neuroreport 17: $19-22$.

Lui S, Deng W, Huang X, Jiang L, Ma X, Chen H et al (2009). Association of cerebral deficits with clinical symptoms in antipsychotic-naive first-episode schizophrenia: an optimized voxel-based morphometry and resting state functional connectivity study. Am J Psychiatry 166: 196-205.

Lui S, Li T, Deng W, Jiang L, Wu Q, Tang H et al (2010). Shortterm effects of antipsychotic treatment on cerebral function in drug-naive first-episode schizophrenia revealed by 'resting state' functional magnetic resonance imaging. Arch Gen Psychiatry 67: 783-792.

Lynall M-E, Bassett DS, Kerwin R, McKenna PJ, Kitzbichler M, Muller U et al (2010). Functional connectivity and brain networks in schizophrenia. J Neurosci 30: 9477-9487.

Ma X, Wang Q, Sham PC, Liu X, Rabe-Hesketh S, Sun X et al (2007). Neurocognitive deficits in first-episode schizophrenic patients and their first-degree relatives. Am J Med Genet Part B Neuropsychiatr Genet 144B: 407-416.

Mesholam-Gately RI, Giuliano AJ, Goff KP, Faraone SV, Seidman LJ (2009). Neurocognition in first-episode schizophrenia: a metaanalytic review. Neuropsychology 23: 315-336.

Notredame C, Pins D, Deneve S, Jardri R (2014). What visual illusions teach us about schizophrenia. Front Integr Neurosci 8: 63.

Orliac F, Naveau M, Joliot M, Delcroix N, Razafimandimby A, Brazo P et al (2013). Links among resting-state default-mode network, salience network, and symptomatology in schizophrenia. Schizophr Res 148: 74-80.

Overall J, Gorham D (1962). The brief psychiatric rating scale. Psychological Reports 10: 799-812.

Patriat R, Molloy EK, Meier TB, Kirk GR, Nair VA, Meyerand ME et al (2013). The effect of resting condition on resting-state fMRI reliability and consistency: a comparison between resting with eyes open, closed, and fixated. Neuroimage 78: 463-473.

Patterson JV, Hetrick WP, Boutros NN, Jin Y, Sandman C, Stern $\mathrm{H}$ et al (2008). P50 sensory gating ratios in schizophrenics and controls: a review and data analysis. Psychiatry Res 158: 226-247. 
Percival D, Walden A (2000). Wavelet Methods for Time Series Analysis (Cambridge Series in Statistical and Probabilistic Mathematics). Cambridge University Press: New York, NY, USA. Pettersson-Yeo W, Allen P, Benetti S, McGuire P, Mechelli A (2011). Dysconnectivity in schizophrenia: where are we now? Neurosci Biobehav Rev 35: 1110-1124.

Power JD, Cohen AL, Nelson SM, Wig GS, Barnes KA, Church JA et al (2011). Functional network organization of the human brain. Neuron 72: 665-678.

Ren W, Lui S, Deng W, Li F, Li M, Huang X et al (2013). Anatomical and functional brain abnormalities in drug-naive first-episode schizophrenia. Am J Psychiatry 170: 1308-1316.

Repovs G, Csernansky JG, Barch DM (2011). Brain network connectivity in individuals with schizophrenia and their siblings. Biol Psychiatry 69: 967-973.

Rodríguez-Sánchez JM, Crespo-Facorro B, González-Blanch C, Perez-Iglesias R, Vázquez-Barquero JLPAFIP Group Study (2007). Cognitive dysfunction in first-episode psychosis: the processing speed hypothesis. Br J Psychiatry Suppl 51: s107-s110.

Roiser JP, Howes OD, Chaddock CA, Joyce EM, McGuire P (2013). Neural and behavioral correlates of aberrant salience in individuals at risk for psychosis. Schizophr Bull 39: 1328-1336.

Seidman LJ, Giuliano AJ, Meyer EC, Addington J, Cadenhead KS, Cannon TD et al (2010). Neuropsychology of the prodrome to psychosis in the NAPLS consortium: relationship to family history and conversion to psychosis. Arch Gen Psychiatry 67: 578-588.

Shepherd AM, Laurens KR, Matheson SL, Carr VJ, Green MJ (2012). Systematic meta-review and quality assessment of the structural brain alterations in schizophrenia. Neurosci Biobehav Rev 36: 1342-1356.

Takeuchi H, Taki Y, Hashizume H, Sassa Y, Nagase T, Nouchi R et al (2011). Effects of training of processing speed on neural systems. J Neurosci 31: 12139-12148.

Tankus A, Fried I (2012). Visuomotor coordination and motor representation by human temporal lobe neurons. J Cogn Neurosci 24: 600-610.
Turken A, Whitfield-Gabrieli S, Bammer R, Baldo JV, Dronkers NF, Gabrieli JDE (2008). Cognitive processing speed and the structure of white matter pathways: convergent evidence from normal variation and lesion studies. Neuroimage 42: 1032-1044.

Turner JA, Damaraju E, van Erp TGM, Mathalon DH, Ford JM, Voyvodic J et al (2013). A multi-site resting state fMRI study on the amplitude of low frequency fluctuations in schizophrenia. Front Neurosci 7: 137.

Umbricht D, Krljes S (2005). Mismatch negativity in schizophrenia: a meta-analysis. Schizophr Res 76: 1-23.

Vagharchakian L, Dehaene-Lambertz G, Pallier C, Dehaene S (2012). A temporal bottleneck in the language comprehension network. J Neurosci 32: 9089-9102.

Wang X, Xia M, Lai Y, Dai Z, Cao Q, Cheng Z et al (2014). Disrupted resting-state functional connectivity in minimally treated chronic schizophrenia. Schizophr Res 156: 150-156.

White T, Ehrlich S, Ho B-C, Manoach DS, Caprihan A, Schulz SC et al (2013). Spatial characteristics of white matter abnormalities in schizophrenia. Schizophr Bull 39: 1077-1086.

Woodward ND, Karbasforoushan H, Heckers S (2012). Thalamocortical dysconnectivity in schizophrenia. Am J Psychiatry 169: 1092-1099.

Xu P, Huang R, Wang J, Van Dam NT, Xie T, Dong Z et al (2014). Different topological organization of human brain functional networks with eyes open versus eyes closed. Neuroimage 90C: 246-255.

Yang GJ, Murray JD, Repovs G, Cole MW, Savic A, Glasser MF et al (2014). Altered global brain signal in schizophrenia. Proc Natl Acad Sci USA 111: 7438-7443.

Zhang D, Guo L, Hu X, Li K, Zhao Q, Liu T (2012). Increased cortico-subcortical functional connectivity in schizophrenia. Brain Imaging Behav 6: 27-35.

Zhou Y, Liang M, Tian L, Wang K, Hao Y, Liu H et al (2007). Functional disintegration in paranoid schizophrenia using resting-state fMRI. Schizophr Res 97: 194-205.

Supplementary Information accompanies the paper on the Neuropsychopharmacology website (http://www.nature.com/npp) 